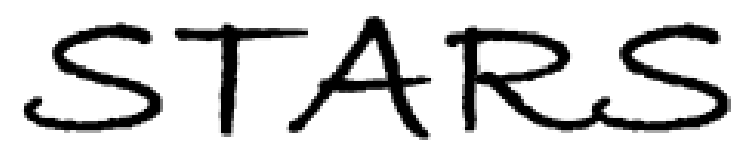

University of Central Florida

STARS

$1-1-2010$

\title{
Superconducting properties of a two-dimensional doped semiconductor
}

\author{
V. M. Loktev \\ V. Turkowski \\ University of Central Florida
}

Find similar works at: https://stars.library.ucf.edu/facultybib2010

University of Central Florida Libraries http://library.ucf.edu

This Article is brought to you for free and open access by the Faculty Bibliography at STARS. It has been accepted for inclusion in Faculty Bibliography 2010 s by an authorized administrator of STARS. For more information, please contact STARS@ucf.edu.

\section{Recommended Citation}

Loktev, V. M. and Turkowski, V., "Superconducting properties of a two-dimensional doped semiconductor" (2010). Faculty Bibliography 2010s. 459.

https://stars.library.ucf.edu/facultybib2010/459

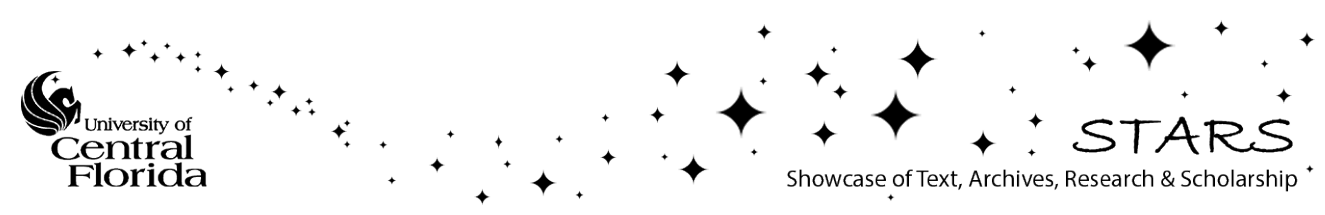




\section{Superconducting properties of a two- dimensional doped semiconductor}

Cite as: Low Temp. Phys. 36, 1004 (2010); https://doi.org/10.1063/1.3521570

Published Online: 10 December 2010

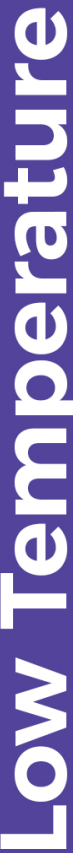

V. M. Loktev, and V. Turkowski

LOW TEMPERATURE TECHNIQUES OP『ICAL CAVITY PHYSICS MITIGATING THERMAL \& VIBRATIONAL NOISE

\section{DOWNLOAD THE WHITE PAPER}




\title{
Superconducting properties of a two-dimensional doped semiconductor
}

\author{
V. M. Loktev \\ Bogolyubov Institute of Theoretical Physics, National Academy of Sciences of Ukraine, $14 b$ Metrologichna \\ Str., Kiev 03680, Ukraine
}

V. Turkowski ${ }^{a)}$

Department of Physics and Nanoscience and Technology Center, University of Central, Florida, Orlando, FL 32816, USA

(Submitted April 12, 2010)

Fiz. Nizk. Temp. 36, 1244-1247 (October-November 2010)

\begin{abstract}
This is a study of the superconducting properties of a two-dimensional model with an additional (insulating) gap $\Delta_{\text {ins }}$ that depends on temperature and doping. In particular, we study the doping dependence of the Berezinskii-Kosterlitz-Thouless critical temperature $T_{c}$ and the superconducting pseudogap temperature $T_{c}^{M F}$ for different values of $\Delta_{\text {ins }}$ by taking hydrodynamic fluctuations of the superconducting order parameter into account. We show that the gap $\Delta_{\text {ins }}$ affects the values of the superconducting gap and the temperatures $T_{c}$ and $T_{c}^{M F}$ within the range of carrier densities where the $\Delta_{\text {ins }}$ approaches zero. In particular, the derivatives of these quantities have a jump in this region. We discuss the possible relevance of these results to high-temperature superconductors. (C) 2010 American Institute of Physics. [doi:10.1063/1.3521570]
\end{abstract}

\section{INTRODUCTION}

Despite more than 20 years of effort, our theoretical understanding of high-temperature superconductors (HTSCs) remains incomplete. One of the crucial tasks is to understand the so-called pseudogap phase, observed in hole-doped cuprates at low carrier densities. In this phase, the quasiparticle density of states has a pseudogap near the Fermi level, even when there is no superconductivity at high temperatures and/or very low doping (see, for example, Ref. 1). In this phase, these materials have many properties which differ from the Fermi-liquid behavior. Usually, two types of scenario are used to explain this phenomenon: the superconducting, or Emery-Kivelson, ${ }^{2}$ scenario and a scenario where an additional nonsuperconducting gap is present. In the first case, the pseudogap is a result of fluctuations in the superconducting order parameter at high temperatures. That is, it is assumed that noncorrelated superconducting pairs are formed below some temperature, which we call the meanfield critical temperature $T_{c}^{M F}$. The order parameters (their phases) become correlated (algebraically ordered) and the system undergoes a superconducting Berezinskii-KosterlitzThouless transition at critical temperature $T_{c}<T_{P G}$. (For a review, see Ref. 3.) The existence of such a pseudogap phase for $T_{c}<T$ has been confirmed experimentally in several cuprates, where the Nernst effect was observed (see, for example, Refs. 4 and 5). However, anomalous properties (the pseudogap phase) are observed in the cuprates at yet higher temperatures than the "Nernst" temperature $T_{0}=T_{c}^{M F}$, which is of order $T_{c}$.

According to the second scenario, there is an additional gap, which is nonzero even in the nonsuperconducting state (see, for example, Ref. 4). The origin of this gap is debated, although many researchers tend to believe that it is defined by the properties of the magnetic subsystem. In this paper, we consider the superconducting properties of a model with a second gap, which we refer to as an insulating gap, $\Delta_{\text {ins }}$. In particular, we study how the magnitude of this gap affects the superconducting properties of the system, such as the doping dependence of the zero-temperature superconducting gap and the critical temperatures $T_{c}$ and $T_{c}^{M F}$. We assume that $\Delta_{\text {ins }}$ depends on the temperature and doping level $\delta$, and that it is zero above the pseudogap temperature curve $T^{*}(\delta)$. The model we use is a more realistic generalization of the model proposed by Nozieres and Pistolesi, ${ }^{6}$ who considered the case of constant $\Delta_{\text {ins }}$. We show that the temperature and doping dependence of $\Delta_{\text {ins }}$ affects the superconducting properties of the system; in particular, it can produce a discontinuity in the derivatives of the gap and temperature with respect to the doping. Experimental observations of such discontinuities may indicate the existence of a second gap. The magnitude of the gap, $\Delta_{\text {ins }}$, can be estimated using a phenomenological model of this type.

We are happy to contribute this paper to the special issue of Low Temperature Physics devoted to the 75th birthday of Professor I. O. Kulik, a world-renowned expert in the fields of weak superconductivity and HTSC.

The paper is organized as follows: the model and the main equations are presented in Sec. II. In Sec. III, we solve the model for different values of the insulating gap and derive a temperature phase diagram of the system. A summary and conclusions are given in Sec. IV.

\section{THE MODEL AND MAIN EQUATIONS}

Here we consider the phenomenological model of Nozieres and Pistolesi for a doped semiconductor with a freeelectron dispersion relation $\xi_{\mathbf{k}}=\varepsilon_{\mathbf{k}}-\mu$, which sets in at $\pm \Delta_{\text {ins }}$ $\left(-W / 2-\Delta_{\text {ins }}<\varepsilon_{\mathbf{k}}<-\Delta_{\text {ins }}\right.$ and $\Delta_{\text {ins }}<\varepsilon_{\mathbf{k}}<W / 2+\Delta_{\text {ins }}$, where $W$ is the free quasiparticle bandwidth and $\mu$ is the chemical potential). We consider a generalized case where $\Delta_{\text {ins }}$ depends on temperature and doping, i.e., 


$$
\begin{aligned}
\Delta_{\text {ins }}(T, \delta)= & \Delta_{\text {ins }}\left[1-\left(\frac{T}{T_{P G}}\right)^{2}-\left(\frac{\delta}{\delta_{\mathrm{cr}}}\right)^{2}\right] \\
& \times \theta\left[1-\left(\frac{T}{T_{P G}}\right)^{2}-\left(\frac{\delta}{\delta_{\text {opt }}}\right)^{2}\right] .
\end{aligned}
$$

This phenomenological dependence is chosen as a semiquantitative model for the doping dependence of the pseudogap temperature which sets in at some magnetic temperature $T_{P G}$ with zero doping and goes to zero at a doping level $\delta_{\text {opt }}$ close to the optimal doping and corresponding to the maximum of $T_{c}$. In HTSCs, the pseudogap temperature $T_{P G}$ is usually defined by the magnetic subsystem. Here we set $T_{P G}=0.03 \mathrm{eV}$ and $\delta_{\mathrm{opt}}=0.165$ in most cases. For simplicity, we also assume that the electron attraction is local with energy $V$. When the hydrodynamic (second order) fluctuations of the phase of the superconducting order parameter are included, the following system of equations is obtained for the superconducting gap $\Delta_{\mathbf{k}}$, chemical potential, and critical temperature $T_{c}$ (see, e.g., Refs. 7-9):

$$
\begin{aligned}
& 1=V \int \frac{d^{2} k}{(2 \pi)^{2}} \gamma_{k}^{2} \frac{\tanh \frac{E_{\mathbf{k}}}{2 T}}{2 E_{\mathbf{k}}}, \\
& \delta=\int \frac{d^{2} k}{(2 \pi)^{2}}\left[1-\tanh \left(\frac{E_{\mathbf{k}}}{2 T}\right) \frac{\xi_{\mathbf{k}}}{E_{\mathbf{k}}}\right], \\
& T=\frac{\pi \delta}{8 m}-\frac{\pi}{32 m^{2}} \frac{1}{T} \int \frac{d^{2} k}{(2 \pi)^{2}} \frac{k^{2}}{\cosh ^{2}\left(E_{\mathbf{k}} / 2 T\right)},
\end{aligned}
$$

where $E_{\mathbf{k}}=\xi_{\mathrm{k} 2}+\Delta_{\mathbf{k} 2}$ is the quasiparticle spectrum in the superconducting state, $\Delta_{\mathbf{k}}=\Delta \gamma_{\mathrm{k}}$ is the Fourier transform of the amplitude of the order parameter, and $\gamma_{\mathbf{k}}$ is the order parameter symmetry factor (equals 1 in the $s-$, and $\cos k x-\cos k y$ in the $d$-wave case, for example). $\delta$ is the doping (carrier density in the system), and $m$ is the quasiparticle mass. In the two dimensional case, the doping is linearly proportional the Fermi energy $\epsilon_{F}=\pi \delta / m$ (for simplicity, in the following we set $\pi / m=1)$.

\section{SUPERCONDUCTING PROPERTIES OF THE MODEL}

\section{A. Zero-temperature limit}

For simplicity we consider the $s$-pairing case. In the zero-temperature limit, the system of equations for the gap and the chemical potential takes the form

$$
\begin{aligned}
& 1=V \int \frac{d^{2} k}{(2 \pi)^{2}} \gamma_{\mathbf{k}}^{2} \frac{1}{2 E_{\mathbf{k}}}, \\
& \delta=\int \frac{d^{2} k}{(2 \pi)^{2}}\left[1-\frac{\xi_{\mathbf{k}}}{E_{\mathbf{k}}}\right],
\end{aligned}
$$

which follows from Eqs. (2) and (3) and can be easily solved (for the square of the dispersion). In particular, in the case with no insulating gap $\left(\Delta_{\text {ins }}=0\right)$ and a wide bandwidth $\mathrm{W}$, the gap and the chemical potential increase monotonically with doping:

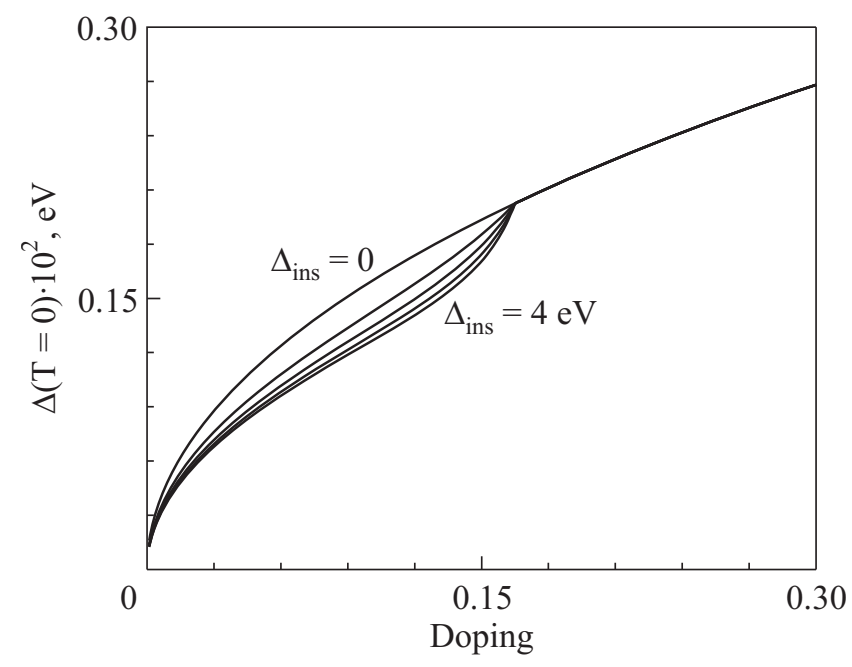

FIG. 1. The doping-dependence of the superconducting gap for $\mathrm{V}$ $=0.15 \mathrm{eV}, \mathrm{W}=4 \mathrm{eV}$ and different values of the gap $\Delta_{\text {ins }}=0,1,2,3$, and $4 \mathrm{eV}$ (from left to right). The discontinuity in the derivative $\partial \Delta / \partial \delta$ at $\delta$ $=\delta_{\text {opt }}=0.165$ corresponds to the critical density when the gap $\Delta_{\text {ins }}$ disappears. Here and in other figures by doping we mean the Fermi energy.

$$
\Delta=\sqrt{2\left|\varepsilon_{b}\right| \epsilon_{F}}, \quad \mu=-\frac{\left|\varepsilon_{b}\right|}{2}+\epsilon_{F},
$$

where $\varepsilon_{b}=-2 W \exp (-4 \pi / m V)$ is the two-particle bound state energy. The chemical potential changes sign at $\epsilon_{F}$ $=\left|\varepsilon_{b}\right| / 2$; thus, a crossover from superfluidity of local boson pairs to superconductivity of Cooper pairs takes place (see, e.g., Ref. 10). A similar situation occurs when the gap $\Delta_{\text {ins }}$ $(T=0)$ is included. Moreover, as shown in Ref. 6, an insulator-superconductor crossover takes place when $\Delta_{\text {ins }}$ $(T=0, \delta=0)$ is equal to one half of the superconducting (BCS) gap in the highly-doped (metallic) case.

In general, when the gap $\Delta_{\text {ins }}$ is doping-independent, it leads to a decrease of the superconducting gap, but the gap does not decrease dramatically. In our case, when

$$
\Delta_{\text {ins }}(0, \delta)=\Delta_{\text {ins }}\left[1-\left(\frac{\delta}{\delta_{\text {opt }}}\right)^{2}\right] \theta\left[1-\left(\frac{\delta}{\delta_{\text {opt }}}\right)^{2}\right],
$$

there is a discontinuity in the derivative of the gap at the critical density $\delta_{\text {opt }}$ (Fig. 1). This effect increases with increasing $\Delta_{\text {ins }}$ and it can be tested experimentally. The discontinuity in the derivative of the gap is a consequence of the discontinuity in the derivative of $\Delta_{\text {ins }}(T=0, \delta)$ at $\delta=\delta_{\text {opt }}$. Therefore, when there is a second gap $\Delta_{\text {ins }}$, the dopingdependence of the superconducting gap must have distinctive behavior near the critical doping.

\section{B. The case of finite temperature}

The variations in the critical temperatures with doping level for different values of the gap $\Delta_{\text {ins }}$ are illustrated in Figs. 2, 3, and 5.

These figures show that this gap affects the temperatures, especially $T_{c}^{M F}$. In the region of nonzero insulating gaps $(\delta$ $<\delta_{\text {opt }}$ ), the mean-field critical temperature is lower than when $\Delta_{\text {ins }}=0$ (Fig. 2).

The decrease in the temperature as the gap increases is not, however, very strong for moderate insulating gaps (on the order of or less than $0.01 \mathrm{eV}$ ). More remarkable is the 


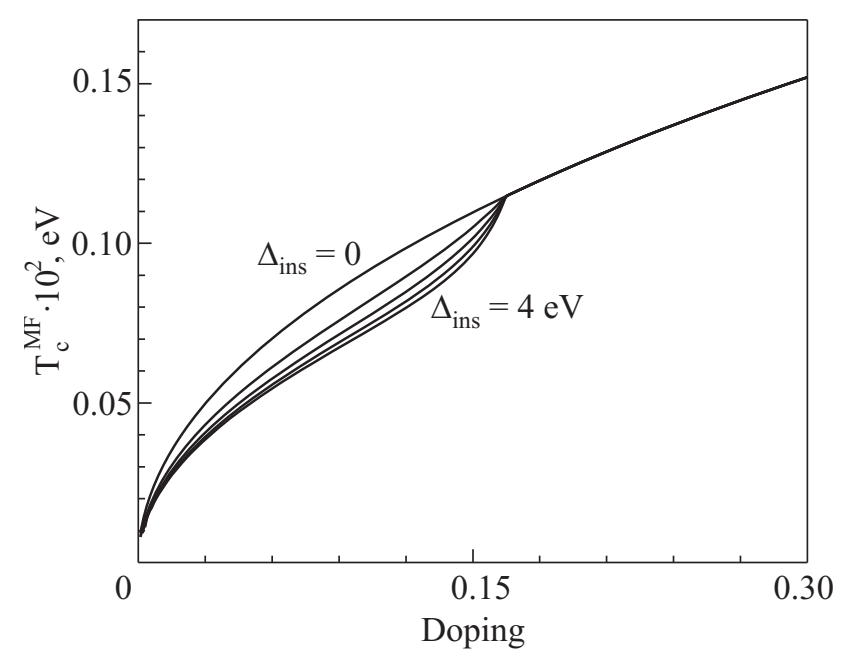

FIG. 2. As in Fig. 1 for the mean-field critical temperature.

fact that the critical temperature has a discontinuity in its derivative near the doping at which $\Delta_{\text {ins }}$ goes to zero. This is a consequence of a discontinuity of this sort in the derivative for $\Delta_{\text {ins }}$ near the optimal doping. We have chosen a doping dependence for the insulating gap of the type in Eq. (1) for which the derivative is a discontinuous function of doping at $\delta_{\text {opt }}$. Whether a doping dependence of this sort for the second gap is realistic is an open question because of the experimental difficulty of measuring it in the region near optimal doping. An extrapolation of an experimental plot of the PG temperature as a function of doping (see Fig. 5) suggests that such a dependence is quite possible. Calculations of the Berezinskii-Kosterlitz-Thouless critical temperature show that this temperature also depends weakly on $\Delta_{\text {ins }}$ (Fig. 3). It is approximately equal to $\epsilon_{F} / 8$ for almost all doping levels. Contrary to the case of $T_{c}^{M F}$, we have found that $T_{c}$ increases slightly with rising $\Delta_{\text {ins }}$. However, the derivative of this temperature with respect to the doping has a large discontinuity near $\delta_{\text {opt }}$ (Fig. 4), which could be measured experimentally. Figure 5 is a typical phase diagram for this model. It shows that the superconducting pseudogap region $\left(T_{c}<T<T_{c}^{M F}\right)$ is quite wide and increases with higher doping. This agrees

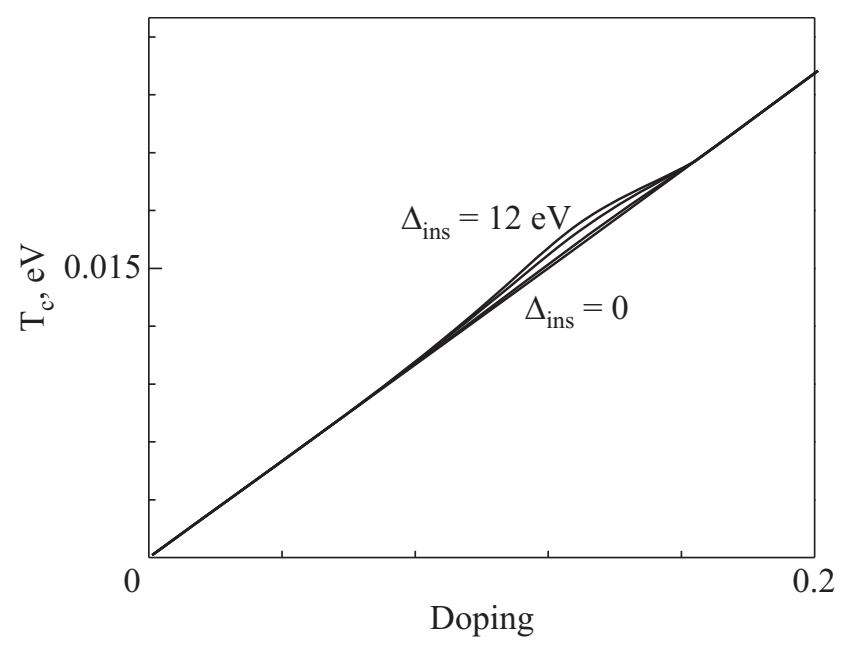

FIG. 3. The doping dependence of the superconducting critical temperature for $\mathrm{V}=0.5 \mathrm{eV}, \mathrm{W}=4 \mathrm{eV}$ and different values of the gap $\Delta_{\mathrm{ins}}=0,5,10$, and $12 \mathrm{eV}$ (from right to left).

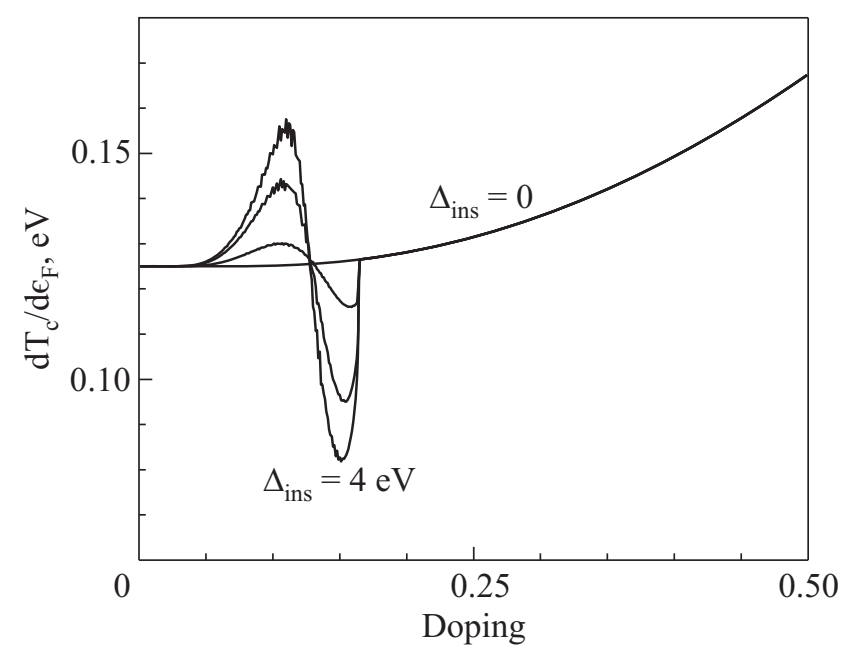

FIG. 4. The derivative of the superconducting critical temperature with respect to the doping as a function of doping. The values of the parameters are as in Fig. 3.

qualitatively with the experiments. We did not find suppression of superconductivity in the over-doped regime in this simple model. This can be obtained by assuming a decrease in the pairing interaction $V$ with higher doping. This situation corresponds, for instance, to decreased spin ordering and spin-wave pairing with higher doping.

\section{CONCLUSIONS}

In this paper we have modelled the superconducting properties of doped semiconductors with a gap that depends on temperature and doping. This dependence has been chosen in a way such that that it can semiquantitatively reproduce the pseudogap phase of cuprate superconductors. In particular, this gap disappears when temperature is higher than the doping dependent PG temperature $T_{P G}$. We did not find dramatic changes in the superconducting gap and critical temperatures with increasing $\Delta_{\text {ins. }}$. However, in the temperature-carrier-density region where $\Delta_{\text {ins }}$ disappears, all

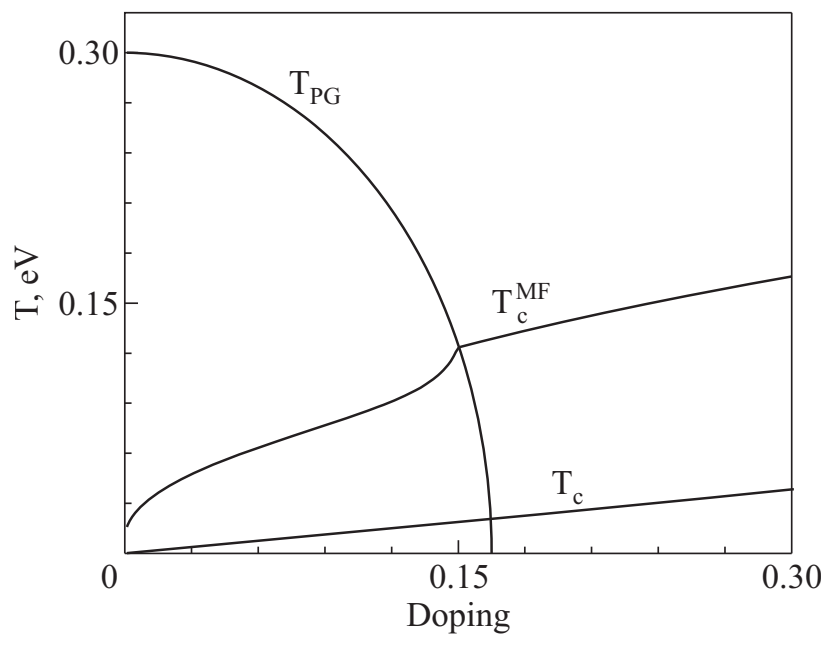

FIG. 5. A temperature-doping phase diagram for the model with two gaps with $\mathrm{V}=0.5 \mathrm{eV}, \mathrm{W}=4 \mathrm{eV}, \mathrm{T}_{\mathrm{PG}}=0.3 \mathrm{eV}$ and $\Delta_{\mathrm{ins}}=4 \mathrm{eV}$. 
the superconducting properties were found to have a change in their doping dependence. For example, the mean-field critical temperature and zero-temperature superconducting gap decrease with increasing $\Delta_{\text {ins }}$ in this region. The critical temperature increases slightly when the doping is close to the critical value. However, its derivative with respect to doping has a large discontinuity in this region, similar to those in the derivatives of the zero-temperature gap and the mean field critical temperature. This is the major result of this paper. Experimental observation of such nonmonotonic variations in the superconducting quantities with doping can indirectly demonstrate the existence of a second gap of this sort in the spectrum, which is often related to the pseudogap in underdoped cuprates. Moreover, with the aid of a simple phenomenological model of this kind one can estimate the possible magnitude of this gap.

V. T. would like to thank R. Klemm for a useful communication and to acknowledge DOE for financial support through grant number DOE-DE-FG02-07ER15842.

a)Email: vturkows@mail.ucf.edu

${ }^{1}$ T. Timusk and B. Statt, Rep. Prog. Phys. 62, 61 (1999).

${ }^{2}$ V. M. Loktev, R. M. Quick, and S. G. Sharapov, Phys. Rep. 349, 2 (2001).

${ }^{3}$ V. J. Emery and S. Kivelson, Nature (London) 374, 434 (1995).

${ }^{4}$ P. A. Lee, N. Nagaosa, and X.-G. Wen, Rev. Mod. Phys. 78, 17 (2006).

${ }^{5}$ Ya. Wang, L. Li, and N. P. Ong, Phys. Rev. B 73, 024510 (2006).

${ }^{6}$ P. Nozieres and F. Pistolesi, Eur. Phys. J. B 10, 649 (1999).

${ }^{7}$ V. P. Gusynin, V. M. Loktev, and S. G. Sharapov, JETP Lett. 65, 182 (1997).

${ }^{8}$ V. M. Loktev, S. G. Sharapov, and V. M. Turkowski, Physica B \& C 296, 84 (1998).

${ }^{9}$ V. M. Loktev and V. M. Turkowski, JETP 87, 329 (1998).

${ }^{10}$ V. M. Loktev and V. M. Turkowski, Fiz. Nizk. Temp. 30, 247 (2004) [Low Temp. Phys. 30, 179 (2004)].

This article was published in English in the original Russian journal. Reproduced here with stylistic changes by AIP. 